\title{
Analysis of cord blood gas at delivery: questionnaire study of practice in the United Kingdom
}

\author{
Jason Waugh, Anthony Johnson, Andrew Farkas
}

A consensus statement from the International Cerebral Palsy Task Force outlined essential criteria to define an acute perinatal hypoxic event; criteria included evidence of a metabolic acidosis in fetal umbilical arterial cord blood or in very early neonatal blood samples $(\mathrm{pH}<7$ and base deficit $\geqslant 12 \mathrm{mmol} / \mathrm{l})$. Pathological fetal acidaemia may be correlated with an increased risk of neurological deficit. ${ }^{2}$

The Royal College of Obstetricians and Gynaecologists and the Royal College of Midwives have jointly stated that "the routine measurement of cord blood gases is essential for all caesarean sections or instrumental deliveries for fetal distress indication and consideration should be given to measurement of cord blood gases following all deliveries. The presence of normal gases, but not $\mathrm{pH}$ alone, largely excludes hypoxia as a cause of brain damage and has important medicolegal implications." 3 We undertook a national survey of current practice and opinions regarding the analysis of cord blood gas at delivery.

\section{Methods and results}

We sent an anonymised questionnaire to the 285 obstetric units in the United Kingdom. Questionnaires (see $B M$ /s website) were completed in accordance with the unit's protocol and returned by November 1999. To maintain anonymity and to encourage all units to respond, no comparison was made between unit size and clinical practice.

A total of 224 units $(79 \%)$ returned the questionnaire. Of these, nine no longer had delivery suites (because of mergers or closures), leaving 215 (75\%) for analysis.

The table shows that $160(74 \%)$ units had a selective policy, that is, sampling some deliveries with specific indications. Of these, 138 (64\%) units analysed cord blood for more than one indication.

Of the 181 units currently sampling cord blood gases, 98 (54\%) sample both arterial and venous blood

Analysis of cord blood gases among obstetric units in the United Kingdom $(n=215)$. Values are numbers (percentages)

\begin{tabular}{lcc} 
Indication for analysis & $\begin{array}{c}\text { Units } \\
\text { currently } \\
\text { performing } \\
\text { analysis }\end{array}$ & $\begin{array}{c}\text { Units that } \\
\text { would perform } \\
\text { analysis if } \\
\text { able to change } \\
\text { policy }\end{array}$ \\
\hline All lower segment caesarean section & $34(16)$ & $26(12)$ \\
\hline All emergency lower segment caesarean section & $97(45)$ & $82(38)$ \\
\hline All instrumental deliveries & $45(21)$ & $63(29)$ \\
\hline All deliveries where fetal distress is suspected & $124(58)$ & $97(45)$ \\
\hline $\begin{array}{l}\text { All deliveries complicated by meconium } \\
\text { stained liquor }\end{array}$ & $44(20)$ & $67(31)$ \\
\hline No deliveries & $34(16)$ & $14(7)$ \\
\hline All deliveries & $21(10)$ & $77(36)$ \\
\hline
\end{tabular}

and $33(18 \%)$ sample venous blood only. Ninety eight (54\%) perform a full blood gas analysis and 43 (24\%) perform a $\mathrm{pH}$ analysis only.

In all, $172(80 \%)$ units recognised that analysis of cord blood gas was clinically useful; 183 (85\%) considered it was useful for audit and teaching purposes and $194(90 \%)$ for medicolegal purposes. Six (3\%) units felt that cord blood gas analysis had no place in obstetric practice despite $34(16 \%)$ units currently not performing the test.

\section{Comment}

Clinical practice regarding the analysis of cord blood gas varies considerably. There is a desire to change practice in many units, and a belief that such analysis has a clinical, teaching, or medicolegal use.

It has been suggested that both arterial and venous samples should be tested to ensure that separate vessels have been sampled. ${ }^{4}$ This suggestion, together with the recommendations of the Royal College of Obstetricians and Gynaecologists and the Royal College of Midwives ${ }^{3}$ concerning a full blood gas analysis, are followed in only $54 \%$ of units.

Recording cord blood gases is important if cerebral palsy is later diagnosed. If the prevalence of cerebral palsy is 2 per 1000 among normally grown infants delivered at full term, in a year a typical obstetric unit (3000 deliveries) will have six cases. About one case will be associated with genuine perinatal asphyxia. Some deliveries will be uncomplicated, without signs of fetal distress, and be under the care of midwifery teams. If it is not routine practice to record analysis of cord blood gas then important evidence of a normal acid-base status at delivery will not be available if the diagnosis of cerebral palsy is made in later childhood. With settlements for such cases now regularly over $£ 3 m$ it may be cost effective to include the recording of cord blood gas analysis in delivery suite budgets.

Contributors: AF and AJ had the original idea for the study. All authors designed the study and questionnaire and jointly wrote the paper. JW analysed the data and is the guarantor.

Funding: None.

Competing interests: None declared.

1 MacLennan A for the International Cerebral Palsy Task Force. A template for defining a causal relationship between acute intrapartum events and cerebral palsy: international consensus statement. $B M J$ 1999;319:1054-9.

2 Goldaber GK, Gilstrap III LC, Leveno KJ, Dax JS, McIntyre DD. Pathologic fetal acidaemia. Obstet Gynaecol 1991;78:1103-11.

3 Royal College of Obstetricians and Gynaecologists, Royal College of Midwives. Towards safer childbirth. Minimum standards for the organisation of labour wards. Report of a joint working party. London: RCOG Press, 1999:22.

4 Westgate J, Garibaldi JM, Green KR. Umbilical cord blood gas analysis at delivery: a time for quality data. Br J Obstet Gynaecol 1994;101: 1054-63.

(Accepted 17 May 2001)
Department of Obstetrics and Gynaecology, Royal Hallamshire Hospital, Sheffield S10 2SF

Jason Waugh specialist registrar Anthony Johnson consultant obstetrician and gynaecologist

Andrew Farkas consultant obstetrician and gynaecologist

Correspondence to: A Farkas farkas@doctors. org.uk

BMJ 2001;323:727

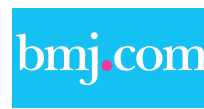

The complete questionnaire is available on the BMJ's website 